\title{
STRONG BANDS OF GROUPS OF LEFT QUOTIENTS
}

\author{
by MIROSLAV ĆIRIĆ and STOJAN BOGDANOVIĆ
}

(Received 11 January, 1995)

1. Introduction and preliminaries. An interesting concept of semigroups (and also rings) of (left) quotients, based on the notion of group inverse in a semigroup, was developed by J. B. Fountain, V. Gould and M. Petrich, in a series of papers (see [5]-[12]). Among the most interesting are semigroups having a semigroup of (left) quotients that is a union of groups. Such semigroups have been widely studied. Recall from [3] that a semigroup has a group of left quotients if and only if it is right reversible and cancellative. A more general result was obtained by V. Gould [10]. She proved that a semigroup has a semilattice of groups as its semigroup of left quotients if and only if it is a semilattice of right reversible, cancellative semigroups. This result has been since generalized by A. El-Qallali [4]. He proved that a semigroup has a left regular band of groups as its semigroup of left quotients if and only if it is a left regular band of right reversible, cancellative semigroups. Moreover, he proved that such semigroups can be also characterised as punched spined products of a left regular band and a semilattice of right reversible, cancellative semigroups. If we consider the proofs of their theorems, we will observe that the principal problem treated there can be formulated in the following way: Given a semigroup $S$ that is a band $B$ of right reversible, cancellative semigroups $S_{i}, i \in B$, to each $S_{i}$ we can associate its group of left quotients $G_{i}$. When is it possible to define a multiplication of $Q=\bigcup_{i \in B} G_{i}$ such that $Q$ becomes a semigroup having $S$ as its left order, and especially, that $Q$ becomes a band $B$ of groups $G_{i}, i \in B$ ? Applying the methods developed in [1] (see also [2]), in the present paper we show how this problem can be solved for $Q$ to become a strong band of groups (that is in fact a band of groups whose idempotents form a subsemigroup, by [16, Theorem 2]. Moreover, we show how Gould's and El-Quallali's constructions of semigroups of left quotients of a semilattice and a left regular band of right reversible, cancellative semigroups, can be simplified.

Throughout this paper, for a semilattice $Y, S=\left(Y ; S_{\alpha}\right)$ will mean that a semigroup $S$ is a semilattice $Y$ of semigroups $S_{\alpha}, \alpha \in Y$. Especially, for a band $B, B=\left(Y ; B_{\alpha}\right)$ will mean that $B$ is a semilattice $Y$ of rectangular bands $B_{\alpha}, \alpha \in Y$ (i.e. $Y$ is the greatest semilattice homomorphic image of $B$ ). For a congruence $\rho, \rho^{\natural}$ will denote its natural homomorphism.

Let $B$ be a band. By $\leq$ we will denote the natural partial order on $B$, i.e. a relation on $B$ defined by: $j \leq i \Leftrightarrow i j=j i=j(i, j \in B)$, and $\leqslant$ will denote a quasi-order on $B$ defined by: $j \leqslant i \Leftrightarrow j=j i j(i, j \in B)$. Clearly, $\leq$ and $\leqslant$ coincide if and only if $B$ is a semilattice. Further, for $i \in B,[i]$ will denote the class of $i$ with respect to the smallest semilattice congruence on $B$. It is easy to verify that $j \leqslant i \Leftrightarrow[j] \leqslant[i]$, for all $i, j \in B$.

Let $B$ be a band. To each $i \in B$ we associate a semigroup $S_{i}$ and an oversemigroup $D_{i}$ of $S_{i}$ such that $D_{i} \cap D_{j}=\varnothing$, if $i \neq j$. For $i, j \in B, i \geqslant j$, let $\phi_{i, j}$ be a mapping of $S_{i}$ into $D_{j}$ and suppose that the family of $\phi_{i, j}$ satisfies the following conditions:

(1) $\phi_{i, i}$ is the identity mapping on $S_{i}$, for each $i \in B$;

Supported by Grant 0401B of RFNS through Math. Inst. SANU.

Glasgow Math. J. 38 (1996) 237-242. 
(2) $\left(S_{i} \phi_{i, i j}\right)\left(S_{j} \phi_{j, i j}\right) \subseteq S_{i j}$, for all $i, j \in B$;

(3) $\left[\left(a \phi_{i, i j}\right)\left(b \phi_{j, i j}\right)\right] \phi_{i j, k}=\left(a \phi_{i, k}\right)\left(b \phi_{j, k}\right)$, for $a \in S_{i}, b \in S_{j}, i j \geqslant k, i, j, k \in B$.

Define a multiplication * on $S=\bigcup_{i \in B} S_{i}$ by:

(4) $a * b=\left(a \phi_{i, i j}\right)\left(b \phi_{j, i j}\right) \quad\left(a \in S_{i}, b \in S_{j}\right)$.

Then $S$ is a band $B$ of semigroups $S_{i}, i \in B$, in notation $S=\left(B ; S_{i}, \phi_{i, j}, D_{i}\right)$ [1]. The symbol " $*$ " will be further omitted. If we assume $i=j$ in (3), then we obtain that $\phi_{i, k}$ is a homomorphism, for all $i, k \in B, i \geqslant k$.

Further, if $D_{i}=S_{i}$, for each $i \in B$, then we write $S=\left(B ; S_{i}, \phi_{i, j}\right)$. Here the condition (2) can be omitted. If $S=\left(B ; S_{i}, \phi_{i, j}\right)$ and if $\left\{\phi_{i, j} \mid i, j \in B, i \geqslant j\right\}$ is a transitive system of homomorphisms, i.e. if $\phi_{i, j} \phi_{j, k}=\phi_{i, k}$, for $i \geqslant j \geqslant k$, then we will write $S=\left[B ; S_{i}, \phi_{i, j}\right]$, and we will say that $S$ is a strong band $B$ of semigroups $S_{i}$. In the case when $B$ is a semilattice, we obtain a strong semilattice of semigroups.

If $P$ and $Q$ are two semigroups with a common homomorphic image $Y$, then a spined product of $P$ and $Q$ with respect to $Y$ is $S=\{(a, b) \in P \times Q \mid a \varphi=b \psi\}$, where $\varphi: P \rightarrow Y$ and $\psi: Q \rightarrow Y$ are homomorphisms onto $Y$. If $P_{\alpha}=\alpha \varphi^{-1}, Q_{\alpha}=\alpha \psi^{-1}, \alpha \in Y$, then $S=\bigcup_{\alpha \in Y} P_{\alpha} \times Q_{\alpha}$. Clearly, $S$ is a subdirect product of $P$ and $Q$. A punched spined product of $P$ and $Q$ with respect to $Y$ is any semigroup isomorphic to some subdirect product of $P$ and $Q$ contained in their spined product with respect to $Y$ [4].

An element $a$ of a semigroup $S$ is completely regular if there exists $x \in S$ such that $a=a x a$ and $a x=x a$. It is well known that $a$ is completely regular if and only if it lies in some subgroup of $S$, so completely regular elements will be also called group elements. If $a$ is completely regular, then there exists a unique $x \in S$ such that $a=a x a, x=x a x$ and $a x=x a$, which is the inverse of $a$ in the maximal subgroup of $S$ containing it, so such an element will be called a group inverse of $a$ and it will be denoted by $a^{-1}$.

An element $a$ of a semigroup $S$ is square-cancellable if, for all $x, y \in S^{1}$,

$$
a^{2} x=a^{2} y \text { implies } a x=a y \text { and } x a^{2}=y a^{2} \text { implies } x a=y a .
$$

Let $S$ be a subsemigroup of a semigroup $Q$. Recall from [9] that $S$ is a left order in $Q$ or that $Q$ is a semigroup of left quotients of $S$ if

(i) every square-cancellable element of $S$ lies in a subgroup of $Q$;

(ii) every element $q$ of $Q$ can be written as $q=a^{-1} b$, for some elements $a, b \in S$.

Clearly, if $Q$ is a union of groups, then the condition (i) can be omitted.

A semigroup $S$ is right reversible if $S a \cap S b \neq \varnothing$, for all $a, b \in S$.

For undefined notions and notation we refer to [3], [13] and [15].

2. The main results. First we will prove the following lemma.

Lemma 1. Let $S=\left(B ; S_{i}, \phi_{i, j}, G_{i}\right)$, and for each $i \in B$, let $S_{i}$ be a right reversible, cancellative semigroup with $G_{i}$ as its group of left quotients. Then, for all $i, j \in B, i \geqslant j, \phi_{i, j}$ can be extended to a homomorphism $\varphi_{i, j}$ of $G_{i}$ into $G_{j}$ such that there exists a $Q=\left[B ; G_{i}, \varphi_{i, j}\right]$.

Proof. Let $\circ$ denote the multiplications in groups $G_{i}, i \in B$. For $i, j \in B, i \geqslant j, \phi_{i, j}$ can be (uniquely) extended to a homomorphism $\varphi_{i, j}$ of $G_{i}$ into $G_{j}$ and then for $a, b \in S_{i}$ we 
have

$$
\left(a^{-1} \circ b\right) \varphi_{i, j}=\left(a \phi_{i, j}\right)^{-1} \circ\left(b \phi_{i, j}\right)
$$

Let us prove that $\left\{\varphi_{i, j} \mid i, j \in B, i \geqslant j\right\}$ is a transitive system of homomorphisms. Since $G_{i}$ is the group of left quotients of $S_{i}$, for any $i \in B$, it is enough to show that $a \varphi_{i, j} \varphi_{j, k}=a \varphi_{i, k}$, for all $i, j, k \in B$ such that $i \geqslant j \geqslant k$ and any $a \in S_{i}$. Assume $x, y \in S_{j}$ such that $a \varphi_{i, j}=x^{-1} \circ y$, i.e. $x \circ\left(a \varphi_{i, j}\right)=y$. Then $y x=y \circ x=x \circ\left(a \varphi_{i, j}\right) \circ x=x \circ\left(a \phi_{i, j}\right) \circ x$. By (3) and (4) it follows that $x a x=x \circ\left(a \phi_{i, j}\right) \circ x$, and hence $y x=x a x$. Again by (3) and (4) we obtain

$$
\left(y \phi_{j, k}\right) \circ\left(x \phi_{j, k}\right)=(y x) \phi_{j, k}=(x a x) \phi_{j, k}=\left(x \phi_{j, k}\right) \circ\left(a \phi_{i, k}\right) \circ\left(x \phi_{j, k}\right),
$$

whence $y \phi_{j, k}=\left(x \phi_{j, k}\right) \circ\left(a \phi_{i, k}\right)$, by the cancellativity in $G_{k}$. Hence,

$$
a \varphi_{i, k}=a \phi_{i, k}=\left(x \phi_{j, k}\right)^{-1} \circ\left(y \phi_{j, k}\right)=\left(x^{-1} \circ y\right) \varphi_{j, k}=a \varphi_{i, j} \varphi_{j, k},
$$

which was to be proved.

Now we go to the main theorem of this paper.

THEOREM 1. The following conditions on a semigroup $S$ are equivalent:

(i) $S$ is a left order in a strong band of groups;

(ii) $S=\left(B ; S_{i}, \phi_{i, j}, G_{i}\right)$, where, for each $i \in B, S_{i}$ is a right reversible, cancellative semigroup with $G_{i}$ as its group of left quotients;

(iii) $S$ is a punched spined product of $a$ band $B=\left(Y ; B_{\alpha}\right)$ and a semigroup $T=\left(Y ; T_{\alpha}\right)$, with respect to a semilattice $Y$, where, for each $\alpha \in Y, T_{\alpha}$ is a right reversible, cancellative semigroup.

Proof. (i) $\Rightarrow$ (ii). This follows immediately by Propositions 2 and 4 of [11].

(ii) $\Rightarrow$ (i). This follows by Lemma 1 .

(ii) $\Rightarrow$ (iii). Let $B=\left(Y ; B_{\alpha}\right)$. By [1, Theorem 2], $S$ is a semilattice $Y$ of semigroups $\left(B_{\alpha} ; S_{i}, \phi_{i, j}, G_{i}\right)$, a relation $\rho$ on $S$ defined by: $a \rho b$ if and only if $a \in S_{i}, b \in S_{j}, i, j \in B$, $[i]=[j]$ and $a \phi_{i, k}=b \phi_{j, k}$, for each $k \in B$ such that $i, j \geqslant k$, is a congruence, $T=S / \rho$ is a semilattice $Y$ of semigroups $T_{\alpha}=S_{\alpha} \rho^{\natural}, \alpha \in Y$, and $S$ is a punched spined product of $B$ and $T$ with respect to $Y$. It remains to prove that for each $\alpha \in Y, T_{\alpha}$ is cancellative and right reversible.

Let $\alpha \in Y$. Assume $u, v, w \in T_{\alpha}$ such that $u w=v w$. Then $u=a \rho^{\natural}, v=b \rho^{\natural}$ and $w=c \rho^{\natural}$, for some $a, b, c \in S_{\alpha}$. Let $a \in S_{i}, b \in S_{j}, c \in S_{k}$, for some $i, j, k \in B_{\alpha}$. Assume $l \in B$ such that $i, j \geqslant l$. Then $k, i k, j k \geqslant l$ and

$$
\begin{aligned}
\left(a \phi_{i, l}\right)\left(c \phi_{k, l}\right) & =\left[\left(a \phi_{i, i k}\right)\left(c \phi_{k, i k}\right)\right] \phi_{i k, l}=(a c) \phi_{i k, l} \\
& =(b c) \phi_{j k, l}=\left[\left(b \phi_{j, j k}\right)\left(c \phi_{k, j k}\right)\right] \phi_{j k, l}=\left(b \phi_{j, l}\right)\left(c \phi_{k, l}\right),
\end{aligned}
$$

since $a c \rho b c$, i.e. $u w=v w$. Now, by the cancellativity in $G_{l}, a \phi_{i, l}=b \phi_{j, l}$. Thus, $a \rho b$, i.e. $u=v$. Hence, $T_{\alpha}$ is right cancellative. Similarly we prove left cancellativity in $T_{\alpha}$.

Let $u, v \in T_{\alpha}$. Then $u=a \rho^{\natural}, v=b \rho^{\natural}$, for some $a, b \in S_{\alpha}$, and $a \in S_{i}, b \in S_{j}$, for some $i, j \in B_{\alpha}$. By Lemma 1 , for all $i, j \in B, i \geqslant j, \phi_{i, j}$ can be extended to a homomorphism $\varphi_{i, j}$ of $G_{i}$ into $G_{j}$ such that there exists a $Q=\left[B ; G_{i}, \varphi_{i, j}\right]$. Now $\left(a \phi_{i, i j}\right)\left(b \phi_{j, i j}\right)^{-1} \in G_{i j}$, so $\left(a \phi_{i, j j}\right)\left(b \phi_{j, i j}\right)^{-1}=x^{-1} y$, for some $x, y \in S_{i j}$, i.e. $x\left(a \phi_{i, i j}\right)=y\left(b \phi_{j, i j}\right)$, whence

$$
y b=y\left(b \phi_{j, i j}\right)=x\left(a \phi_{i, i j}\right)=(x a) \phi_{i j i, i j} .
$$


Assume $k \in B$ such that $i j, i j i \geqslant k$. Then

$$
(y b) \phi_{i j, k}=(x a) \phi_{i j i, i j} \phi_{i j, k}=(x a) \varphi_{i j i, i j} \varphi_{i j, k}=(x a) \varphi_{i j i, k}=(x a) \phi_{i j i, k} .
$$

Therefore, $y b \rho x a$, whence $\left(x \rho^{\natural}\right) u=\left(y \rho^{\natural}\right) v$, so $T_{\alpha}$ is right reversible.

(iii) $\Rightarrow$ (i). Without loss of generality we can assume that $S \subseteq B \times T$, i.e. $S \subseteq \bigcup_{\alpha \in Y} B_{\alpha} \times T_{\alpha}$. By [10, Theorem 3.1], $T$ is a left order in a semigroup $Q$, where $Q=\left(Y ; G_{\alpha}\right)$ and for each $\alpha \in Y, G_{\alpha}$ is a group, and also, for each $\alpha \in Y, G_{\alpha}$ is a group of left quotients of $T_{\alpha}$. Let $P$ be the spined product of $B$ and $Q$ with respect to $Y$, i.e. let $P=\bigcup_{\alpha \in Y} B_{\alpha} \times G_{\alpha}$. By [16, Theorem 4] (see also [14, Theorem 3.2]), $P$ is a strong band of groups. It remains to prove that $P$ is a semigroup of left quotients of $S$. Assume an arbitrary $(i, a) \in P$. Then $i \in B_{\alpha}, a \in G_{\alpha}$, for some $\alpha \in Y$. Since $S$ is a subdirect product of $B$ and $T$, there exists $b \in T$ such that $(i, b) \in S$, and hence $b \in T_{\alpha}$. Thus, $b a^{-1} \in G_{\alpha}$, so $b a^{-1}=x^{-1} y$, for some $x, y \in T_{\alpha}$, and further, there exists $j, k \in B$ such that $(j, x)$, $(k, y) \in S$. Now $j, k \in B_{\alpha}$, so $(i, b x b)=(i, b)(j, x)(i, b) \in S,(i k, b y)=(i, b)(k, y) \in S$, and (by $)^{-1} b x b=y^{-1} b^{-1} b x b=y^{-1} x b=\left(b a^{-1}\right)^{-1} b=a b^{-1} b=a$, whence

$$
(i, a)=\left(i,(b y)^{-1} b x b\right)=(i k, b y)^{-1}(i, b x b) .
$$

Therefore, $P$ is a semigroup of left quotients of $S$.

Semigroups having a rectangular group of (left) quotients have been considered by several authors. By Theorem 1 we obtain the following corollary.

COROLLARY 1. The following conditions on a semigroup $S$ are equivalent:

(i) $S$ is a left order in a rectangular group;

(ii) $S=\left(B ; S_{i}, \phi_{i, j}, G_{i}\right)$, where $B$ is a rectangular band and for each $i \in B, S_{i}$ is a right reversible, cancellative semigroup with $G_{i}$ as its group of left quotients;

(iii) $S$ is a subdirect product of a rectangular band and a right reversible, cancellative semigroup.

Finally, the next theorem, together with Lemma 1, shows how Gould's and El-Qallali's constructions of semigroups of left quotients of a semilattice and a left regular band of right reversible, cancellative semigroups, can be simplified.

THEOREM 2. Let $S$ be a left regular band $B$ of right reversible, cancellative semigroups $S_{i}$, $i \in B$, and for each $i \in B$, let $G_{i}$ be the group of left quotients of $S_{i}$. Then $S=\left(B ; S_{i}, \phi_{i, j}, G_{i}\right)$.

Proof. Let $\circ$ denote the multiplications in groups $G_{i}, i \in B$, and let $\left\{u_{i} \mid i \in B\right\} \subseteq S$ such that $u_{i} \in S_{i}$, for each $i \in B$. For $i, j \in B, i \geqslant j$, define a mapping $\phi_{i, j}: S_{i} \rightarrow G_{j}$ by:

$$
a \phi_{i, j}=u_{j}^{-1} \circ\left(u_{j} a\right) \quad\left(a \in S_{i}\right) .
$$

Since $B$ is a left regular band, then $u_{j} a \in S_{j}$, so $u_{j}^{-1} \circ\left(u_{j} a\right) \in G_{j}$. Clearly (1) holds. Assume 
$i, j \in B, a \in S_{i}, b \in S_{j i}$. Further, since $B$ is a left regular band, then $u_{i j}, u_{i j} a \in S_{i j}$, so $v u_{i j} a=w u_{i j}$, for some $v, w \in S_{i j}$. Now

$$
\begin{aligned}
& \left(a \phi_{i, i j}\right) \circ\left(b \phi_{j, i j}\right)=u_{i j}^{-1} \circ\left(u_{i j} a\right) \circ u_{i j}^{-1} \circ\left(u_{i j} b\right) \\
& \quad=u_{i j}^{-1} \circ v^{-1} \circ v \circ\left(u_{i j} a\right) \circ u_{i j}^{-1} \circ\left(u_{i j} b\right)=u_{i j}^{-1} \circ v^{-1} \circ w \circ u_{i j} \circ u_{i j}^{-1} \circ\left(u_{i j} b\right) \\
& \quad=u_{i j}^{-1} \circ v^{-1} \circ w \circ\left(u_{i j} b\right)=u_{i j}^{-1} \circ v^{-1} \circ\left(w u_{i j} b\right)=u_{i j}^{-1} \circ v^{-1} \circ\left(v u_{i j} a b\right) \\
& \quad=u_{i j}^{-1} \circ v^{-1} \circ v \circ\left(u_{i j} a b\right)=u_{i j}^{-1} \circ\left(u_{i j} a b\right)=u_{i j}^{-1} \circ u_{i j} \circ(a b)=a b .
\end{aligned}
$$

Therefore, (2) and (4) hold.

Assume $i, j, k \in B, i j \geqslant k, a \in S_{i}, b \in S_{j}$. Since $B$ is a left regular band, then $u_{k} a, u_{k} \in S_{k}$, whence $v u_{k} a=w u_{k}$, for some $v, w \in S_{k}$. Now

$$
\begin{aligned}
& {\left[\left(a \phi_{i, i j}\right) \circ\left(b \phi_{j, i j}\right)\right] \phi_{i j, k}=(a b) \phi_{i j, k}=u_{k}^{-1} \circ\left(u_{k} a b\right)=u_{k}^{-1} \circ v^{-1} \circ\left(v u_{k} a b\right)} \\
& \quad=u_{k}^{-1} \circ v^{-1} \circ\left(w u_{k} b\right)=u_{k}^{-1} \circ v^{-1} \circ w \circ\left(u_{k} b\right) \\
& \quad=u_{k}^{-1} \circ v^{-1} \circ w \circ u_{k} \circ u_{k}^{-1} \circ\left(u_{k} b\right)=u_{k}^{-1} \circ v^{-1} \circ v \circ\left(u_{k} a\right) \circ u_{k}^{-1} \circ\left(u_{k} b\right) \\
& \quad=u_{k}^{-1} \circ\left(u_{k} a\right) \circ u_{k}^{-1} \circ\left(u_{k} b\right)=\left(a \phi_{i, k}\right) \circ\left(b \phi_{j, k}\right) .
\end{aligned}
$$

Therefore, $S=\left(B ; S_{i}, \phi_{i, j}, G_{i}\right)$.

ACKNOWLEDGEMENTs. The authors are indebted to the referee for several useful comments and suggestions concerning the presentation of this paper.

\section{REFERENCES}

1. M. Ćirić and S. Bogdanović, Spined products of some semigroups, Proc. Japan Acad. Ser. A Math. Sci. 69 (1993), 357-362.

2. M. Ćirić and $\mathbf{S}$. Bogdanović, Subdirect products of a band and a semigroup, to appear.

3. A. H. Clifford and G. B. Preston, The algebraic theory of semigroups, vol I (American Mathematical Society, 1961). $29-40$.

4. A. El-Qallali, Left regular bands of groups of left quotients, Glasgow Math. J. 33 (1991),

5. J. B. Fountain and V. Gould, Completely 0 -simple semigroups of quotients. II. Contributions to general algebra, 3 (Hölder-Pichler-Tempsky, 1985), 115-124.

6. J. B. Fountain and M. Petrich, Brandt semigroups of quotients, Math. Proc. Cambridge Philos. Soc. 98 (1985), 413-426.

7. J. B. Fountain and M. Petrich, Completely 0-simple semigroups of quotients, J. Algebra 101 (1986), 365-402.

8. J. B. Fountain and M. Petrich, Completely 0-simple semigroups of quotients. III, Math. Proc. Cambridge Philos. Soc. 105 (1989), 263-275.

9. V. Gould, Bisimple inverse $\omega$-semigroups of left quotients, Proc. London. Math. Soc. (3) 52 (1986), $95-118$.

10. V. Gould, Clifford semigroups of left quotients, Glasgow Math. J. 28 (1986), 181-191.

11. V. Gould, Orders in semigroups, Contributions to general algebra, 5 (Hölder-PichlerTempsky, 1987), 163-169.

12. V. Gould, Semigroups of left quotients-the uniqueness problem, Proc. Edinburgh Math. Soc. 35 (1992), 213-226.

13. J. M. Howie, An introduction to semigroup theory (Academic Press, 1976).

14. M. Petrich, Regular semigroups which are subdirect products of a band and a semilattice of groups, Glasgow Math. J. 14 (1973), 27-49. 
15. M. Petrich, Introduction to semigroups (Merill, 1973).

16. B. M. Schein, Bands of monoids, Acta Sci. Math. (Szeged) 36 (1974), 145-154.

17. M. Yamada, Strictly inversive semigroups, Bull. Shimane Univ. 13 (1964), 128-138.

UNIVERSITY OF NIక̌

FACULTY OF PHILOSOPHY

Department of Mathematics

18000 Niš, ĆiRILA I MetodiJa 2

YUgosLAVIA
UNIVERSITY OF NIŠ

FACULTY OF ECONOMICS 18000 Niš, TRG JNA 11

YugosLaVIa 